\title{
RECUPERAÇÃO DE OÓCITOS E PRODUÇÃO IN VITRODE EMBRIÕES DE VACAS ESTIMULADAS COM FSH OU ECG
}

\author{
OOCYTE RECOVERY AND IN VITRO PRODUCTION FROM COWS STIMULATED \\ WITH EITHER FSH OR ECG
}

\author{
Ribeiro, L.V.P. ${ }^{1 *}$, Rigolon, L.P. ${ }^{1}$, Cavalieri, F.L.B. ${ }^{2}$, Seko M.B. ${ }^{2}$, Martinez, A.C. ${ }^{1}$, Ribeiro, M.G. ${ }^{1}$, \\ Martins, R.R. ${ }^{1}$, Ávila, M.R. ${ }^{1}$ e De Conti, J.B. ${ }^{1}$
}

'Universidade Estadual de Maringá. Umuarama-Paraná. Brasil. *luvet20@hotmail.com

${ }^{2}$ Centro Universitário de Maringá. Maringá-Paraná. Brasil.

\section{PalaVRas ChaVe ADICIONAIS}

OPU. FIV. Reprodução animal.

\section{RESUMO}

Avaliou-se a recuperação de oócitos e produção de embriões in vitro de 42 vacas, mestiça Nelore proveniente de grupo genético homogêneo, com idade de 4 a 9 anos, com peso médio de 420 $\mathrm{kg}$, estimuladas com FSH ou eCG. Estas foram distribuídas em três grupos: grupo 1, controle $(n=14)$, apenas OPU, grupo 2, tratadas com 1400 UI de eCG, em dose única seguida de aspiração folicular (OPU) $(n=14)$; e, grupo 3 , tratadas com $120 \mathrm{UI}$ de FSH, administrados com intervalo de 12 horas em quatro doses seguida de OPU $(n=14)$. Todos os grupos receberam implante auricular contendo $3 \mathrm{mg}$ de Norgestomet no primeiro dia (D0) associado a administração de $2 \mathrm{mg}$ de benzoato de estradiol via intramuscular. No sétimo dia (D7) foram retirados todos os implantes e na sequência foram realizadas as aspirações ovarianas das vacas do grupo 1. O grupo 2 recebeu a aplicação de eCG no quinto dia e a OPU foi realizada no D7. Já o grupo 3 recebeu o tratamento com FSH no quinto e sexto dia, e foram aspiradas em D7. O procedimento de aspiração folicular foi realizado via transvaginal guiada por ultrassom. Foram realizadas 42 aspirações com obtenção de 627 oócitos, sendo 502 viáveis e 125 inviáveis. Realizou-se a maturação e fecundação in vitro. Avaliaram-se as taxas de clivagem, blastocisto e eclosão. Não houve diferenças significativas $(p>0,05)$ nos parâmetros avaliados entre os grupos. Conclui-se que o estímulo ovariano com FSH ou com eCG nas doses utilizadas, foram insuficientes para incrementar o número e a qualidade de oócitos viáveis

\author{
AdDITIONAL KEYWORDS \\ OPU. FIV. Animal reproduction.
}

submetidos a fertilização in vitro para produção de embriões.

\section{SUMMARY}

It was evaluated the oocyte and embryo in vitro production of 42 Nellore mixed breed cows (Bos taurus indicus) come from de same genetic group, aging from 4 to 9 years, with mean body weight of $420 \mathrm{~kg}$, stimulated with FSH and eCG. The animals were distributed into three groups: group 1 , control $(n=14)$, only with follicular aspiration (OPU); group 2, treated with $1400 \mathrm{UI}$ of eCG in a single dose plus OPU $(n=14)$; and group 3, treated with $120 \mathrm{UI}$ of FSH administered four times plus OPU with $12 \mathrm{~h}$ of interval $(n=14)$. All groups received auricular implant with $3 \mathrm{mg}$ of Norgestomet on the first day (D0) associated with $2 \mathrm{mg}$ of estradiol benzoate intramuscularly. At the seventh day (D7) the implants were removed and the ovarian aspirations were realized on the cows of group 1(OPU). Group 2 had the eCG applied at the fifth day and the OPU was performed at D7. The animals from group 3 were treated with FSH at fifth and sixth day and the aspiration occurred at D7 (OPU). The follicular aspiration procedure was done via transvaginal guided with ultrasound. It was performed 42 aspirations obtaining 627 oocytes, where 502 were viable and 125 nonviable. Then, it was realized the in vitro maturation and fecundation. The cleavage, blastocyst and eclosion rate were evaluated. There were no 
significant difference $(p>0.05)$ at these parameters between the groups. In conclusion, the gonadotrophic stimulation with FSH and eCG, at the doses used in this study, was insufficient to increase the quality of the viable oocytes submitted to in vitro fertilization to produce embryos.

\section{INTRODUÇÃO}

A utilização de fármacos na reprodução animal assistida teve significativos progressos na última década, de modo que, atualmente, alguns ainda vêm sendo intensamente testados e diversos outros já são utilizados rotineiramente. Utilizando-se a hormonioterapia é possível incrementar os índices reprodutivos dos rebanhos de corte e de leite, visando o tratamento de afecções ovarianas ou nos programas de inseminação artificial e superestimulação ovariana em animais de elevado valor genético (Kozicki et al., 2005).

A biotecnologia reprodutiva tem sido utilizada para acelerar e aprimorar a genética de rebanhos, favorecendo difusão de genes de animais com alto valor zootécnico e comercial. O sucesso da transferência de embriões (TE) e da fertilização in vitro (FIV) depende, em grande parte, da resposta à superovulação. Nos atuais programas comerciais de TE, a resposta de doadoras submetidas à superovulação se caracteriza por uma alta variação nas taxas de ovulação e fecundação, o que leva a resultados não tão previsíveis e nem confiáveis na produção de embriões (Boland et al., 1991). A variabilidade na produção de embriões pode ser influenciada por fatores relacionados com o tratamento superovulatório, mas em maior grau por fatores individuais associados às características da dinâmica folicular ovariana (Bó et al., 1995; Madureira e Baruselli, 2000) ou a condição ovariana no momento da superovulação (Monniaux et al., 1983). Técnicas como maturação, fecundação e cultivo in vitro estão sendo utilizadas com o intuito de aumentar o uso de oócitos presentes nos ovários dos animais potencialmente doadores.
Entre as técnicas utilizadas para a coleta de oócitos, a aspiração transvaginal guiada por ultrassom é a que apresenta maior número de embriões produzidos in vitro por doadoras devido a alta repetibilidade, possibilitando maior recuperação de oócitos (Pieterse et al., 1988).

Para Brogliatti e Adams (1996), a superestimulação hormonal aumenta o número de folículos de tamanho adequado para a aspiração e, consequentemente, o número de embriões que podem ser produzidos, devido a maiores taxas de recuperação, porém outros autores utilizando os mesmos tratamentos encontraram respostas diferentes.

Nos últimos anos muitos grupos de pesquisa vêm trabalhando com o intuito de aprimorar a maturação citoplasmática in vitro e, consequentemente, melhorar a capacidade a partir de desenvolvimento embrionário de oócitos provenientes de folículos antrais pequenos. Estudos da regulação da maturação de oócitos são essenciais para a geração de conhecimentos necessários para o aumento dos índices de produção de embriões in vitro.

Hoje a aspiração folicular(OPU), no Brasil, tem sido utilizada com sucesso por instituições dedicadas a pesquisa, assim como pela iniciativa privada na recuperação de oócitos para produção in vitro de embriões bovinos.

O presente trabalho foi desenvolvido com o objetivo de avaliar oócitos de doadoras Nelore mestiças, verificando o efeito do tratamento estimulatório, com dosagem utilizada em programa de superovulação tradicional, com FSH ou eCG sobre a taxa de recuperação, a produção e a qualidade morfológica de oócitos colhidos por aspiração folicular por via transvaginal, orientada pela ultrassonografia e, a subsequente produção in vitro (PIV) de embriões.

\section{MATERIALEMÉTODOS}

O experimento foi desenvolvido na 
Fazenda do CESUMAR (Centro de Ensino Superior de Maringá), no Centro de Biotecnologia em Reprodução Animal, BIOTEC, que dispõe de laboratório de produção in vitro de embriões, localizada no município de Maringá, PR.

Foram utilizadas 42 fêmeas Nelore mestiças, proveniente de um mesmo grupo genético, com idade de 4 a 9 anos, com peso médio de $420 \mathrm{~kg}$. Estas fêmeas foram everminadas com Ivermectina $1 \%$ (Ivomec, Merial $(\mathbb{B})$ e passaram por um período de adaptação de 45 dias, permaneceram em pastagem de Cynodon spp. (grama estrela)

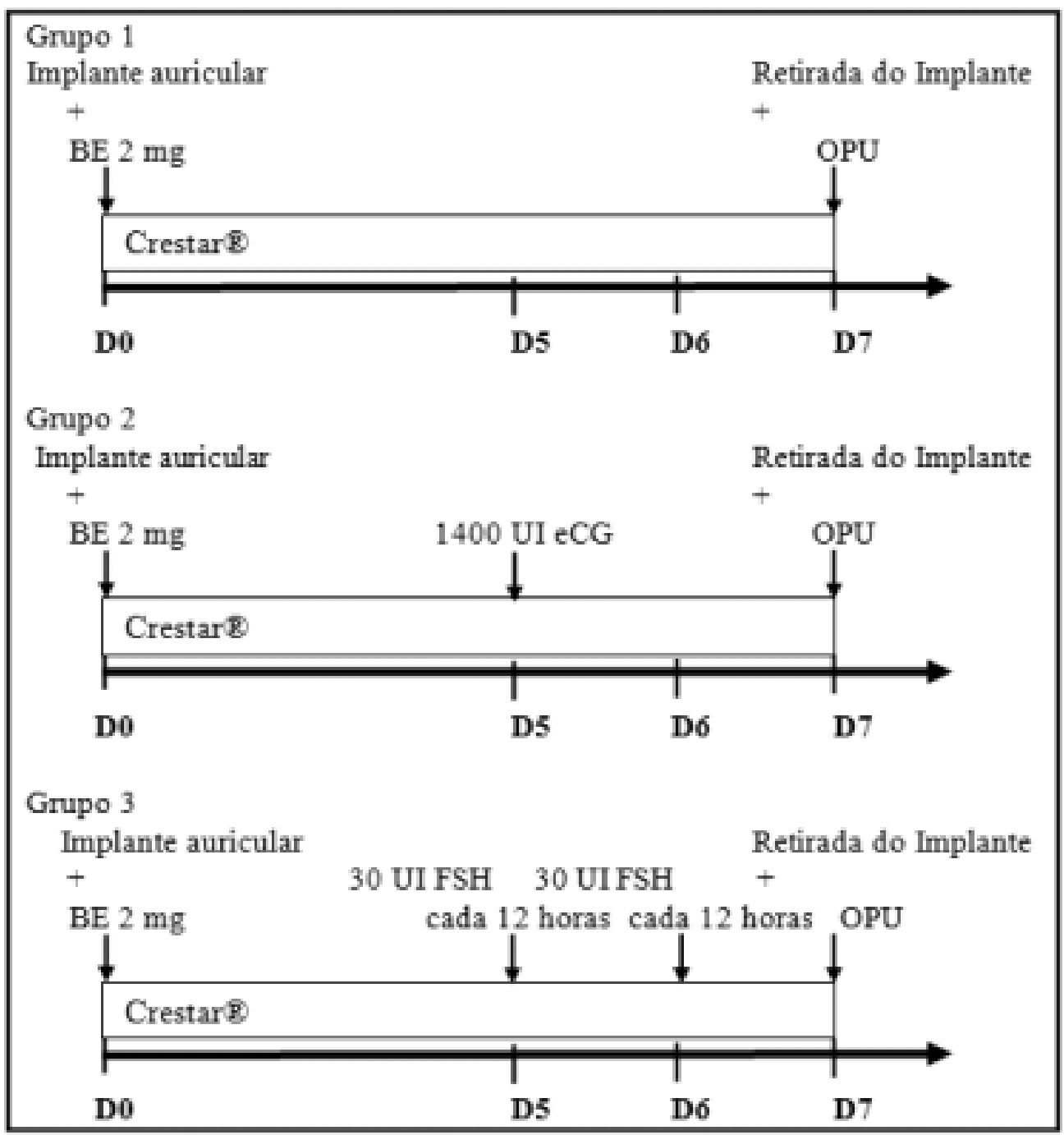

Figura 1. Diagrama esquemático dos protocolos de estimulação e sincronização de ondas foliculares de vacas Nelore mestiças de 4 a 9 anos de idade. (Diagram of follicular wave stimulation and synchronization in 4 to 9 -year-old crossbred Nellore cows). 
e tiveram acesso à água e sal mineral $a d$ libitum.

Todos os animais receberam implante auricular, substância contendo $3 \mathrm{mg}$ de Norgestomet no dia 0 (D0) (Crestar, Intervet, Bosmeer, Holanda), associado a aplicação de $2 \mathrm{mg}$ de benzoato de estradiol (BE) (Cronibest, Biogenesis, Bagó, Curitiba, Paraná), via intramuscular.

As fêmeas foram separadas aleatoriamente, em três grupos de 14 animais: grupo 1: controle, não receberam tratamento estimulatório, apenas OPU em D7; grupo 2: estimulação com 1400 UI de gonodotrofina coriônica equina (eCG), (Folligon ${ }^{\circledR}$, Intervet), via intramuscular, em D5 e OPU em D7; e grupo 3: estimulação com 120 UI de hormônio folículo estimulante (FSH) (Pluset ${ }^{\circledR}$, I.F. Serono, Roma, Itália), administrados via intramuscular em 4 aplicações com 12 horas de intervalo (30 UI), iniciando a partir do quinto dia da colocação do implante auricular, seguida de OPU em D7.

Os implantes foram retirados em sete dias, em todos os animais e, em seguida foi realizada uma aspiração nos animais de cada grupo, totalizando 42 OPUs. O detalhamento do esquema de hormonioterapia dos grupos 1, 2 e 3 estão detalhados na figura 1 .

O procedimento de aspiração folicular foi realizado com ultrassom Aloka SSD-500, acoplado a um transdutor microconvexo de $5 \mathrm{mHz}$ (UST 974-5), sendo que o mesmo foi adaptado a uma guia de aspiração específica para o sistema reprodutor de bovinos. Uma agulha $20 \mathrm{G}$ (WTA $®)$ foi conectada ao sistema de aspiração (Cook VBOA 18L) com tubo Falcon de $50 \mathrm{ml}$. A pressão de vácuo foi obtida com uma bomba Cook V-MAR 5000, ajustada para 38 a $45 \mathrm{mmHg}$, permitindo um fluxo de $12 \mathrm{ml}$ de meio/minuto.

Para inibir os movimentos peristálticos e desconforto ao animal foi feita uma anestesia epidural sacrococcígea, utilizando-se 100 mg de lidocaína a 2\% (Anestésico Pearson $₫$ ) e, em seguida, o transdutor foi inserido até o fundo vaginal e, com o auxílio da manipulação transretal, os ovários foram posicionados para obtenção de uma boa visualização dos folículos na tela do ultrassom.

Os folículos a serem aspirados foram posicionados no percurso da linha de punção indicada na tela do ultrassom e quando se aproximou a agulha do folículo a ser aspirado, foi pressionado o pedal da bomba de vácuo e o oócito aspirado, procedimento repetido em todos os folículos acima de $3 \mathrm{~mm}$ de cada ovário. O meio utilizado para a aspiração, lavagem da agulha e do tubo Falcon receptor dos oócitos foi composto por uma solução de: $2,0 \%$ de soro fetal bovino (Nutricell), 98,0\% de DMPBSFLUSH (Nutricell) e $25 \mathrm{UI} / \mathrm{ml}$ de heparina sódica (Liquemine ${ }^{\circledR}$ ), evitando a coagulação sanguínea no interior do sistema de aspiração.

O material aspirado foi transferido para o filtro de colheita de embriões (EmCom ${ }^{\circledR}$ ) e lavado com a mesma solução utilizada na aspiração. O sedimento restante no filtro foi observado em placas de Petri e efetuado a busca e contagem dos oócitos com posterior classificação da qualidade. Os oócitos foram quantificados e classificados de acordo com sua morfologia (número de camadas de células do cumulus e aspecto do citoplasma) em qualidade 1, 2 e 3 (cumulus completo, parcial ou expandido, respectivamente), oócitos sem cumullus (s/c) ou desnudos, expandidos (exp), degenerados (deg) ou atrésicos (atr), conforme descrito por Gonçalves et al. (2001). Os oócitos considerados viáveis, qualidade 1,2 e 3 , foram lavados em solução comercial de MIV-T (Nutricell) e transportados para o laboratório, em criotubos (Corning $\left.{ }^{\circledR}\right)$, contendo meio de maturação em banho-maria a $35^{\circ} \mathrm{C}$.

No laboratório os oócitos foram lavados três vezes em meio de lavagem TCM-199 com sais de Earles, glutamina e $\mathrm{NaHCO}^{3}$, suplementado com $10 \%$ de soro fetal bovino (SFB), $22 \mu \mathrm{g} / \mathrm{ml}$ piruvato, $50 \mu \mathrm{g} / \mathrm{ml}$ de gentamicina, $0,5 \mu \mathrm{g}$ de FSH$/ \mathrm{ml}, 50 \mu \mathrm{g}$ de LH/ $\mathrm{ml} \mathrm{e} 1 \mu \mathrm{g}$ de estradiol $/ \mathrm{ml}$, mantidos em estufa, a $38,5^{\circ} \mathrm{C}, 5 \%$ de $\mathrm{CO}_{2}$ em ar com máxima 


\section{RECUPERAÇÃO DE OÓCITOS E PRODUÇÃO IN VITRO DE EMBRIÕES}

umidade, durante 22-24 horas. Durante esse período os complexos cumullus oócitos (CCOs) permaneceram em microgotas de $100 \mu \mathrm{l}$ de meio de maturação coberta por óleo mineral.

Os oócitos maturados foram lavados três vezes em $100 \mu \mathrm{l}$ de meio TALP-FIV suplementado com $10 \mu \mathrm{g} / \mathrm{ml}$ de heparina, 22 $\mu \mathrm{l} / \mathrm{ml}$ de piruvato, $50 \mu \mathrm{g} / \mathrm{ml}$ de gentamicina, albumina sérica bovina-BSA (sem ácidos graxos), solução de PHE ( $2 \mu \mathrm{M}$ de penicilina, $1 \mu \mathrm{M}$ de hipotaurina e $0,25 \mu \mathrm{M}$ de epinefrina). O sêmen utilizado foi de uma mesma partida de um touro da raça Nelore, descongelado em banho-maria a $35^{\circ} \mathrm{C}$. Para seleção dos espermatozoides móveis e remoção de diluidores e plasma seminal, foi realizado centrifugação em gradiente percoll $90 \%$ e $45 \%$, a 1200 rotações por minuto, durante 20 minutos. Foi utilizada a concentração de 2 x 106 espermatozoides/ml e estes foram transferidos para microgotas com 20 oócitos/gota juntamente com COCs, onde permaneceram por 18 horas, a $38,5^{\circ} \mathrm{C}$, em atmosfera com $5 \%$ de $\mathrm{CO}_{2}$ em ar.

Após a fecundação, os zigotos foram cultivados in vitro no meio SOF suplementado com $10 \%$ de soro fetal bovino (SFB), com monocamada de células da granulosa. O cultivo foi realizado por 18 horas pósinseminação, em incubadora, com atmosfera gasosa contendo $5 \%$ de $\mathrm{CO}_{2}$, em ar com máxima umidade. Decorridas 48 horas, foi avaliada a taxa de clivagem e feito a renovação do meio de cultivo. Sete dias após a fecundação foram realizadas as avaliações dos embriões de cada grupo, classificando-os em blastocisto inicial, blastocisto, blastocisto expandido ou blastocisto eclodido, conforme classificação recomendada pela Sociedade Internacional de Transferência de Embriões (IETS).

Os dados foram analisados no software SAS Versão 8. A diferença entre os grupos no número de oócitos viáveis, inviáveis, clivados, embriões ou embriões eclodidos foram analisados por análise de variância (PROC GLM) e as diferenças entre médias pelo teste Tukey.

\section{RESULTADOSEDISCUSSÃO}

Para a recuperação de oócitos dos animais dos três tratamentos foram realizadas 42 aspirações foliculares (OPU) em que foram obtidos 627 oócitos, sendo 502 viáveis e 125 inviáveis.

Na tabela I, estão descritos os resultados da aplicação do eCG ou do FSH sobre a qualidade dos oócitos viáveis.

Observou-se que não houve efeito ( $p>0,05)$, dos tratamentos com eCG ou FSH

Tabela I. Resultados médios da aplicação da eCG (gonadotrofina coriônica equina) ou do FSH (hormônio folículo estimulante) sobre a qualidade dos oócitos viáveis de vacas Nelore mestiças de 4 a 9 anos de idade. (Average result of eCG (equine chorionic gonadotropin) or FSH (follicle stimulating hormone) application on the quality of viable oocytes from Nellore crossbred cows from 4 to 9 years of age).

\begin{tabular}{lccc}
\hline & Controle & $\begin{array}{c}\text { Tratamentos } \\
\text { ECG }(1400 \text { UI) }\end{array}$ & FSH (120 UI) \\
\hline Oócitos viáveis & $10,50 \pm 11,56$ & $16,38 \pm 15,21$ & $10,35 \pm 7,27$ \\
Oócitos qualidade 1 & $2,00 \pm 3,96$ & $5,46 \pm 9,00$ & $3,00 \pm 3,74$ \\
Oócitos qualidade 2 & $1,07 \pm 1,59$ & $2,00 \pm 4,16$ & $2,07 \pm 2,05$ \\
Oócitos qualidade 3 & $5,71 \pm 8,58$ & $5,15 \pm 4,91$ & $2,00 \pm 2,07$ \\
Oócitos expandidos & $1,71 \pm 2,26$ & $3,77 \pm 3,58$ & $3,07 \pm 4,68$ \\
\hline
\end{tabular}

${ }^{1}$ Valores não significativos pelo teste de Tukey a $5 \%$. 
Tabela II. Resultados médios da aplicação do eCG (gonadotrofina coriônica equina) ou do FSH (hormônio folículo estimulante) sobre a produção de oócitos inviáveis de vacas Nelore mestiças de 4 a 9 anos. (Average result of eCG (equine chorionic gonadotropin) or FSH (follicle stimulating hormone) application on the production of viable oocytes from Nellore crossbred cows from 4 to 9 years old).

\begin{tabular}{lccr}
\hline & Controle & $\begin{array}{c}\text { Tratamentos } \\
\text { ECG }(1400 \text { UI })\end{array}$ & FSH (120 UI) \\
\hline Oócitos inviáveis & $2,57 \pm 5,54$ & $2,38 \pm 3,50$ & $1,21 \pm 3,11$ \\
Oócitos atrésicos & $1,71 \pm 4,74$ & $4,61 \pm 6,45$ & $1,00 \pm 2,66$ \\
Oócitos degenerado & $0,85 \pm 1,40$ & $0,30 \pm 0,63$ & $0,14 \pm 0,53$ \\
Oócitos desnudo & $0,21 \pm 0,58$ & $0,38 \pm 0,76$ & $0,07 \pm 0,26$ \\
\hline
\end{tabular}

\Valores não significativos pelo teste de Tukey a $5 \%$.

no número de oócitos viáveis com relação ao grupo controle. O número de oócitos viáveis é de importância fundamental em programas de fertilização in vitro (FIV). O fato de não haver diferença significativa entre as quantidades de oócitos morfologicamente viáveis, pode indicar que a sincronização da onda folicular permitiu que todos os oócitos estivessem na mesma fase fisiológica, não havendo diferença entre os estimulados ou não.

Na tabela II, estão expressos os resultados da aplicação do eCG ou do FSH sobre a produção de oócitos inviáveis de vacas Nelore mestiças.

Neste trabalho utilizou-se a préestimulação com FSH ou eCG, após sincronização das ondas foliculares com implante auricular de Norgestomet e aplicação intramuscular de $2 \mathrm{mg}$ de benzoato de estradiol, com intuito de minimizar o efeito de algum folículo dominante (Bó et al., 1995), que resultou em uma média de $10,357,27$ oócitos viáveis com $\mathrm{FSH}$, de 16,38 15,21 , com eCG e de 10,50 11,56 no grupo controle. As diferenças nos resultados encontrados neste trabalho não foram significativas $(\mathrm{p}>0,05)$.

Sendag et al. (2008) encontraram melhores resultados utilizando $500 \mathrm{UI}$ de FSH comparando com a aplicação de 3000 UI de
eCG, em vacas Holandesas. No trabalho os autores sugerem que a resposta ovariana, o número de folículo nos ovários e número oócitos, além de sua qualidade são afetados pelo tipo de gonadotrofina aplicada, sendo o FSH a melhor alternativa para OPU em relação ao eCG. Merton et al. (2003) também encontraram um efeito positivo na préestimulação ovariana com FSH, antes da OPU e, em relação a competência de desenvolvimento dos oócitos. O esquema de superovulação varia em relação à quantidade a ser aplicada, o número de aplicações realizadas e o intervalo entre a última aplicação de FSH e a aspiração. Nibart et al. (1995) indicam que a recuperação de oócitos viáveis pode dobrar com a utilização da superestimulação com FSH. Uma das possíveis explicações para o bom resultado da pré-estimulação ovariana com FSH seria um aumento no número de folículos médios e grandes, impedindo os mesmos de entrarem em atresia, isto poderia resultar em oócitos mais capacitados para sofrerem a maturação in vitro. Stubbings e Walton (1995) relatam que o pré-tratamento com $\mathrm{FSH}$ resulta numa resposta ovariana imediata, produzindo um maior número de folículos para aspiração na primeira semana de tratamento, comparando com animais não estimulados hormonalmente. 


\section{RECUPERAÇÃO DE OÓCITOS E PRODUÇÃO IN VITRO DE EMBRIÕES}

Rouillier et al. (1996); Goodhand et al. (1999); Roover et al. (2005) comentam que o tratamento com FSH supera os resultados obtidos nos tratamento como eCG, sendo para estes autores o método de escolha para superovulação de bovinos. Na maioria dos estudos comparando os dois procedimentos, o tratamento com FSH resultou em leve aumento de número de embriões utilizáveis.

Wang et al. (1988) relataram que o número total de oócitos aumenta com a utilização de eCG, mas o número e percentual de clivagem diminui com altas doses de eCG. Entretanto, esses efeitos negativos não foram observados com doses crescentes de um extrato de pituitária suína altamente purificada (FSH) (Abdul et al., 1989). A dose total de eCG e a sua origem ou método de purificação, são fatores que podem ter contribuído para essas diferenças. Pieterse e Kappen (1992) utilizando um tratamento com eCG em 10 vacas, obtiveram resultados positivos em comparação aos animais não tratados.

Na tabela III, está descrito os resultados médios da aplicação do eCG ou do FSH sobre a produção de embriões in vitro a partir de oócitos aspirados de vacas Nelore mestiças.

Ramos et al. (2006) avaliaram os efeitos de dois protocolos de punção folicular na quantidade/qualidade dos oócitos e na produção de embriões in vitro, em vacas da raça Gir, não lactantes, sendo um grupo sem estimulação hormonal e outro com aplicação de 250 UI de FSH em doses decrescentes. Encontraram taxa de clivagem de $56 \%$ e de blastocisto de $18 \%$, tendo a pré-estimulação ovariana melhorado a qualidade e a taxa de clivagem dos oócitos recuperados por punção folicular. Goodhand et al. (1996) verificaram que o FSH em doses múltiplas e aspiração de animais estimulados uma vez por semana têm sido mais eficientes que uma só administração. Entretanto Monteiro et al. (2009) utilizaram vacas controle, préestimuladas com FSH e OPU 6 horas após e, pré-estimuladas com FSH e OPU 48 após, não tendo ocorrido qualquer diferença. As taxas de clivagem e de blastocisto foram semelhantes entre os três tratamentos, sendo $77,4 \%$ e $42,70 \%, 75,54 \%$ e $31,65 \%, 63,52 \%$ e $33,33 \%$, respectivamente, contudo a taxa de blastocistos eclodidos foi superior nos controles $(30,27 \%)$ em relação aos demais. No trabalho em questão obteve-se resultado semelhante comparando o grupo controle (sem estimulação) e o grupo 3 (tratado com FSH), já que as taxas de clivagem, de blastocisto e de eclosão de todos os grupos foram semelhantes. Satrapa et al. (2005)

Tabela III. Resultado médio e desvio padrão da aplicação do eCG (gonadotrofina coriônica equina) ou do FSH (hormônio folículo estimulante) sobre a produção in vitro de embriões obtidos de aspiração de vacas Nelore mestiças de 4 a 9 anos de idade. (Average results and standard deviation of eCG (equine chorionic gonadotropin) or FSH (follicle stimulating hormone) application on the in vitro production of embryos obtained from aspiration of Nellore crossbred cows).

\begin{tabular}{lccc}
\hline & Controle & Tratamentos & \\
& eCG & FSH \\
\hline Número médio de oócitos enviados a PIV & $10,50 \pm 11,56$ & $16,38 \pm 15,21$ & $10,35 \pm 7,27$ \\
Taxa de clivagem (\%) & $72,57 \pm 33,80$ & $72,73 \pm 28,08$ & $63,35 \pm 37,29$ \\
Taxa de blastocisto (\%) & $37,50 \pm 25,88$ & $36,46 \pm 24,37$ & $38,57 \pm 31,63$ \\
Taxa de eclosão (\%) & $28,07 \pm 24,66$ & $27,92 \pm 25,43$ & $24,58 \pm 27,61$ \\
\hline
\end{tabular}

${ }^{1}$ Valores não significativos pelo teste de Tukey a $5 \%$. 
notaram que a superestimulação ovariana com OPU 48 horas após a última aplicação de FSH não aumentou a produção in vitro de embriões de vacas da raça Nelore, além de as maiores taxas de blastocisto eclodido foram observadas em oócitos provenientes de vacas que não foram superestimuladas. Já, Nonato et al. (2005) observaram que a administração prévia de FSH na aspiração folicular não aumentou a média de oócitos obtidos, porém, aumentou a taxa de produção embrionária, o que é benéfico para a tecnologia de produção de embriões in vitro, discordando dos resultados encontrados no trabalho aqui desenvolvido.

Chaubal et al. (2007) administraram FSH em dose única e múltiplas na estimulação ovariana antes da OPU e, notaram que a administração de FSH em três aplicações no período de 24 horas aumentou, significativamente, a resposta folicular e a recuperação de oócitos em vacas.

Roover et al. (2008) afirmou que os resultados de sete anos de superestimulação ovariana com FSH antes da OPU, não ter melhorado a produção de embriões, mas os oócitos aspirados de vacas superestimuladas apresentaram melhor qualidade.

No presente trabalho foi observada elevada variação na quantidade de oócitos, sendo que no controle foi de 1 a 43, nos pré-

\section{BIBLIOGRAFIA}

Abdul, S.S., Hutchinson, J.S.M., Broadbent, P.J. and Dolman, D.F. 1989. Hormonal profiles in superovulated Hereford $x$ British Friesian heifers. Theriogenology, 31: 253.

Bó, G.A., Adams, G.P., Caccia, M., Martínez, M., Pierson, R.A. and Mapletoft, R.J. 1995. Ovarian follicular wave emergence after treatment with progestagen and estradiol in cattle. Anim. Reprod. Sci., 39: 139-204.

Boland, M.P., Goulding, D. and Roche, J.F. 1991. Alternatives gonadotrophins for superovulation in cattle. Theriogenology, 35: 5-17.

Brogliatti, G.M. and Adams, G.P. 1996. Ultrasound guided transvaginal oocyte collection in estimulados com FSH de 2 a 27 e nos préestimulados com eCG de 2 a 49, o que evidencia diferenças no potencial de vacas utilizadas como doadoras de oócitos. Tal fato torna necessária a avaliação prévia do animal antes de sua utilização em programas comerciais de produção de embriões in vitro e as razões para essa variabilidade ainda são pouco compreendidas (Ramos, 2006).

Considerando as informações concluise que pesquisas de ordem técnica e biológica poderiam contribuir para o incremento da OPU, aumentando a eficiência do procedimento e disponibilizando mais oócitos para os procedimentos de produção in vitro de embriões.

O resultado na recuperação de oócitos após aspiração folicular está condicionado a fatores ainda não totalmente compreendidos, portanto há interesse em realizar estudos tendendo a encontrar condições ótimas para cada situação.

\section{CONCLUSÕES}

O estímulo ovariano com 120 UI de FSH por animal ou com 1400 UI de eCG foi insuficiente para incrementar o número e a qualidade de oócitos viáveis na aspiração folicular transvaginal guiada por ultrassom, não resultando em melhoria na produção de embriões in vitro.

prepupertal calves. Theriogenology, 45: 1163. Chaubal, S.A., Ferre, L.B., Molina, J.A., Faber, D.C., Bols, P.E.J., Rezamand, P., Tian, X. and Yang, X. 2007. Hormonal treatments for increasing the oocyte and embryo production in an OPU-IVP system. Theriogenology, 67: 719728.

Gonçalves, P.B.D., Figueiredo, J.R. e Freitas, V.J.F. 2001. Biotécnicas aplicadas à reprodução animal. Varela Editora e Livraria Ltda. São Paulo. pp. 195-226.

Goodhand, K.L., Watt, R.G., Staines, M.E., Hutchinson, J.S. and Broadbent, P.J. 1999. In vivo oocyte recovery and in vitro embryo 


\section{RECUPERAÇÃO DE OÓCITOS E PRODUÇÃO IN VITRO DE EMBRIÕES}

production from bovine donors aspirated at different frequencies or following FSH treatment. Theriogenology, 51: 951-961.

Goodhand, K.L., Broadbent, P.J., Hutchinson, R. and Watt, G. 1996. In vivo oocyte recovery and in vitro embryo production in cattle pretreated with $\mathrm{FSH}$, progestogen and estradiol. Theriogenology, 45: 355.

Kozicki, L.E., Segui, M.S., Fantini Filho, J.C., Prado, F.R.A., Matté, F., Glaser, J.R.P. e Weiss, R.R. 2005. A somatotrofina bovina (BST) e sua relação com o recrutamento folicular ovariano durante o ciclo estral de vacas. Arch. Vet. Sci., 10: 35-44.

Madureira, E.H e Baruselli, P.S. 2000. Controle farmacológico do ciclo estral em ruminantes. Em: Bó, G.A., G.P. Adams e R.J. Mapletoft. Dinâmica folicular ovárica em bovino. Funvet. São Paulo. pp. 12-34.

Merton, J.S., De Roos, A.P.W., Mullaart, E., De Ruigh, L., Kaal, L., Vos, P.L.A.M. and Dieleman, S.J. 2003. Factors affecting oocyte quality and quantity in commercial application of embryo technologies in the cattle breeding industry. Theriogenology, 59: 651-667.

Monniaux, D., Chupin, D. and Saumande, J. 1983. Superovulatory response of cattle. Theriogenology, 19: 55-81.

Monteiro, F.M, Ferreira, M.M.G., Potiens, J.R., Eberhardt, B.G., Trinca, L.A. and Barros, C.M. 2009. Influence of superovulatory protocols on in vitro production of Nellore (Bos indicus) embryos. Reprod. Dom. Anim., 44. http:// www3.interscience.wiley.com/journal/ 122299747/abstract\#relatedArticles (01/05/ 09).

Nonato Jr., I., Pontes, J.H.F., Ereno Jr., J.C., Sanches, B.V. e Seneda, M.M. 2005. Obtenção de oócitos e produção in vitro de embriões em vacas Nelores (Bos taurus indicus) tratadas com FSH previamente à aspiração. Acta Sci. Vet., 33: 369.

Pieterse, M.C. and Kappen, K.A. 1992. Aspiration of bovine oocytes during transvaginal ultrasound scanning of the ovaries. Theriogenology, 30: 762.

Pieterse, M.C., Kappen, K.A., Kruip, A.M. and
Taverne, M.A.M. 1988. Aspiration of bovine oocytes during transvaginal ultrasound scanning ovaries. Theriogenology, 30: 751756.

Ramos, A.A., Ferreira, A.M., Sá, W.F., Camargo, L.S.A., Viana, J.H.M. e Henry, M.R.J.M. 2006. Protocolos de produção in vitro de embriões na raça Gir. Arq. Bras. Med. Vet. Zootecn., 58: 341347.

Roover, R., Genicot, G., Leonard, S., Bols, P. and Dessy, F. 2005. Ovum pick up and in vitro embryo production in cows superstimulated with an individually adapted superstimulation protocol. Anim. Reprod. Sci., 86: 13-25.

Roover, R., Feugang, J.M., Bols, P.E., Genicot, G. and Hanzen, C.H. 2008. Effects of ovum pick-up frequency and FSH stimulation: a retrospective study on seven years of beef cattle in vitro embryoproduction. Reprod. Dom. Anim., 43: 239-245.

Rouillier, P., Guilbault, L.A., Lussier, J.G. and Matton, P. 1996. Changes in morphological appearance and functional capacity of recruited follicles in cows treated with FSH in the presence or absence of a dominant follicle. Theriogenology, 46: 1053-1061.

Sendag, S, Cetin, Y., Alan, M., Hadeler, K. and Niemann, H. 2008. Effects of eCG and FSH on ovarian response, recovery rate and number and quality of oocytes obtained by ovum pickup in Holstein cows. Anim. Reprod. Sci., 106: 208-214.

Satrapa, R.A., Ferreira, M.M.G., Monteiro, F.M., Ederhardt, B.G., Melo, D.S., Potiens, J.R. e Barros, C.M. 2005. Influência de protocolos de super-estimulação e privação hormonal na produção in vitro de embriões Nelore (Bos taurus indicus). Acta Sci. Vet., 33: 404-404.

Stubbings, R.B. and Walton, J.S. 1995. Effect of ultrasonically guided follicle aspiration on estrous cycle and follicular dynamics in Holstein cows. Theriogenology, 43: 705-712.

Wang, H., Wu, M., Patt, D., Murphy, B.D. and Mapletoft, R.J. 1988. Superovulation in beef heifers with PMSG: effect of dose and monoclonal antibodies to PMSG. Theriogenology, 29: 322. 